\title{
Frequent Hemodialysis Fistula Infectious Complications
}

\author{
Charmaine E. Lok ${ }^{a}$ Jessica M. Sontrop ${ }^{b}$ Rose Faratro ${ }^{a}$ \\ Christopher T. Chan ${ }^{\mathrm{a}}$ Deborah Lynn Zimmerman ${ }^{\mathrm{c}}$ \\ a Division of Nephrology, Department of Medicine, Toronto General Hospital and \\ University of Toronto, Toronto, Ont., b Department of Epidemiology and Biostatistics, \\ Western University, London, Ont., and ' Division of Nephrology, Kidney Research Centre, \\ Ottawa Hospital Research Institute, Ottawa, Ont., Canada
}

\section{Key Words}

Cannulation · Fistula $\cdot$ Infection $\cdot$ Hemodialysis

\begin{abstract}
Background: Few studies have examined if infectious arteriovenous access complications vary with the cannulation technique and whether this is modified by dialysis frequency. We compared the infection rate between fistulas cannulated using buttonhole versus stepladder techniques for patients treated with short daily (SDH) or nocturnal hemodialysis at home (NHD). We also compared patients receiving conventional intermittent hemodialysis (CIHD) using stepladder cannulation. Methods: Data were prospectively collected from 631 patients dialyzed with a fistula from 2001 to 2010 (Toronto and Ottawa, Canada). We compared the person-time incidence rate of bacteremia and local fistula infections using the exact binomial test. Results: Forty-six (7.3\%) patients received SDH ( $\geq 5$ sessions/week, 2-4 h/session), 128 (20.3\%) NHD ( $\geq 4$ sessions/week, $\geq 5$ h/session) and 457 (72\%) CIHD (3 sessions/week, $\leq 4 \mathrm{~h} /$ session). Fifty percent of SDH and $72 \%$ of NHD patients used the buttonhole technique. There were 39 buttonhole-related bacteremias (rate: 0.196/1,000 fistula days) and at least 2 local buttonhole site infections. Staphylococcus aureus accounted for $85 \%$ of the bacteremias. There were 5 (13\%) infection-related hospitalizations and 3 (10\%) serious metastatic infections, including fistula loss. In comparison, there was 1 possible fistula-related infection in CIHD during follow-up (rate: 0.002/1,000 fistula days). Conclusions: The rate of buttonholerelated infections was high among patients on frequent hemodialysis and more than 50 times greater than that among patients on CIHD with the stepladder technique. Most bacteremias were due to $S$. aureus - with serious consequences. The risks and benefits of buttonhole cannulation require individual consideration with careful monitoring, prophylaxis and management.


Lok et al.: Frequent Hemodialysis Fistula Infectious Complications

\section{Introduction}

Once functional, hemodialysis arteriovenous fistulas demonstrate longer cumulative patency as well as fewer infections and complications compared with synthetic arteriovenous grafts and central venous catheters [1-3]. For these reasons, published guidelines recommend the fistula as the preferred hemodialysis access [4-8]. However, fistula cannulation requires more technical skill than graft cannulation [9-11], and nurses rank difficult cannulation as their main concern in hemodialysis [12]. As such, cannulation remains a major barrier to increasing fistula use among dialysis patients $[11,13]$.

Improving cannulation techniques is crucial for improving fistula success and for patients' quality of life. Although the cannulation technique is critical for establishing reliable access to the circulation for hemodialysis, few studies have evaluated if and how dialysis outcomes vary with the cannulation technique. Further, while frequent hemodialysis is associated with improved outcomes compared with conventional intermittent hemodialysis (CIHD), the impact of frequent fistula cannulation on infectious complications is largely unknown and may differ across cannulation techniques.

The two most common cannulation techniques are stepladder and buttonhole cannulation. Stepladder cannulation involves rotating the cannulation sites up and down the length of the access; however, this technique can be problematic for patients with short fistula lengths and for patients in whom the fistula is difficult to cannulate due to aberrant anatomy such as a significant aneurysm. Buttonhole cannulation, originally described by Twardowski [14], is gaining in popularity, especially for home hemodialysis. In buttonhole cannulation, two needles are inserted at the same two spots, at the same angle, at the same depth, every time. This 'constant-site' method is recommended by some to prevent the development of aneurysms and stenosis which sometimes occur with stepladder cannulation if the cannulation area becomes restricted [15-17]. Additional advantages of buttonhole cannulation are purported to include improved ease and speed of cannulation with fewer 'missed sticks', less pain, fewer hematomas and aneurysms as well as faster hemostasis after needle removal [18-23]. In addition, buttonhole cannulation may be preferred by self-cannulators (particularly for home hemodialysis) and when there are limited viable cannulation sites $[15,18]$.

Despite these potential benefits, several studies have reported an elevated rate of infectious complications for buttonhole compared with stepladder cannulation. Studies evaluating in-center transitions to buttonhole cannulation show a marked increase in infections and a significantly increased rate of septic access events [24]. The increased rate of infections seen with buttonhole cannulation is attributed to improper preparation and/or cannulation, especially of nonhealed skin. This issue may be exacerbated as more patients adopt frequent hemodialysis. Whereas CIHD involves 3 sessions/week ( 318 needle insertions/year), frequent hemodialysis, either by short daily (SDH) or nocturnal hemodialysis (NHD), is typically received 4-6 times/week, which amounts to $~ 520$ needle insertions/year [25]. More frequent cannulation and manipulation of the vascular access, as required for frequent hemodialysis, may increase the risk of infectious complications [24].

In this study, we compare the rate of infections between patients performing frequent hemodialysis (SDH or NHD) cannulating their fistulas using the buttonhole technique and those using the stepladder technique. We also evaluate infection rates among patients receiving frequent hemodialysis using buttonhole cannulation and compare them with those among patients receiving CIHD using stepladder cannulation. 


\section{Subjects and Methods}

\section{Study Design and Patient Population}

We conducted a cohort study of adult hemodialysis patients who were dialyzed with an arteriovenous fistula at the University Health Network-Toronto General Hospital (UHN-TGH; Toronto, Ont., Canada) and the Ottawa Hospital Home Dialysis (OHHD; Ottawa, Ont., Canada) unit from January 1, 2000, through December 31, 2010.

Patient information was entered prospectively into a vascular access database by trained data entry staff, and the database was managed by a multidisciplinary vascular access team. Clinical variables were regularly verified by the vascular access coordinator and/or the vascular access program director. The database contains information on patient demographics, the cause of end-stage kidney failure, comorbidities and vascular access treatment trajectories including the type and date of each access creation, cannulation technique, dates and reasons for access failure, details of access monitoring and surveillance, vascular accessrelated radiological interventions and surgeries as well as infectious and noninfectious complications. This study was approved by each site's Institutional Review Board.

\section{Cannulation Procedure}

Prior to cannulation, the dialysis access team evaluated the fistula for maturity and suitability for cannulation. At the UHN-TGH, all in-center CIHD patients ( 3 sessions/week, $\leq 4 \mathrm{~h}$ / session) were cannulated by hemodialysis nurses, while those who received NHD $(\geq 4$ sessions/week, $\geq 5 \mathrm{~h} /$ session) self-cannulated at home. At the OHHD unit, $>90 \%$ of all patients self-cannulated; patients who did not self-cannulate at the OHHD unit were excluded. All cannulators followed a standardized protocol. The buttonhole and stepladder cannulation techniques are described below. All home hemodialysis patients had the option to use buttonhole cannulation with the exception of those with cardiac valve replacement or previous central stenosis that was felt to increase pressure within the access, which were contraindications to buttonhole use in the OHHD program.

\section{Buttonhole Cannulation}

The buttonhole track is created over an average 2-week period (typically 8-10 sessions) using sharp needles and is performed by home hemodialysis nurses, all experienced in creating buttonhole tracks. Usually, 1-2 nurses will establish a track. Once the track is created, blunt-needle cannulation is performed at least twice consecutively by the same vascular access nurse. If successful, subsequent cannulations can be performed by the patient or different nursing staff using a blunt needle. In selected cases in the OHHD program, patients are taught to establish the buttonhole tracks themselves. If there are difficulties placing a blunt needle into an established buttonhole, a conventional sharp needle is used. At least 1 arterial and 1 venous buttonhole are created for each patient. At the UHN-TGH, 2 sets of buttonholes may be created depending on the fistula length, while in the OHHD program, only 1 set of buttonholes is usually created, regardless of the frequency of dialysis treatment.

Before each cannulation, the patient washes the access area thoroughly with an antiseptic solution for 2-5 min (povidone iodine or chlorhexidine $2 \%$ - without alcohol, depending on patient skin sensitivities). Following this, the scab is removed very carefully with a sharp 18-gauge needle or buttonhole pick. Patients who have severe skin sensitivity to povidone iodine or chlorhexidine may clean the area with antibacterial soap and then 'soak' the scab with an alcohol swab and gently lift it off with that gauze. The access area is cleaned using similar cleansing agents as previously described. Patients and nurses are instructed to allow at least $30 \mathrm{~s}$ of contact with the disinfectant before cannulation. Finally, the cannulation is performed using blunt needles (bevel up), maintaining an aseptic technique and following the 
angle of the existing track. If the blunt needle does not slip easily into the track, cannulators are directed to readjust the angle and move up or down on top of the vessel until the correct angle to access the vessel is located.

Stepladder Cannulation

In stepladder cannulation, needle placement is moved sequentially along the length of the fistula at every dialysis session after appropriate skin preparation as discussed above. Needles are placed at least 1 inch apart to avoid previous sites and prevent blood recirculation during dialysis. The patient is taught about the importance of site rotation and instructed to remind the cannulator about the rotation plan.

\section{Measures and Definitions}

Infectious Events

Infectious events were fistula-related bacteremias or local cannulation site infections. Bacteremia was defined by 2 positive blood cultures separated by at least $15 \mathrm{~min}$ and local signs of infection at the cannulation site or along the fistula or if no other alternative source of bacteremia was found. Local fistula infection was defined by nonallergic erythema, pain or tenderness close to the cannulation sites, necrotic scabs or drainage from the cannulation site(s). Bacteremia caused by Staphylococcus aureus or S. epidermidis was considered an infectious event originating from the fistula, even without local signs, if no alternative source (such as a diabetic foot) was identified. Infectious events that recurred in the same patient were counted as new events if they occurred more than 3 weeks after the completion of antibiotic therapy or if a different causal microorganism was identified.

Hemodialysis Modality

Hemodialysis regimens were defined as CIHD (3 sessions/week, $\leq 4 \mathrm{~h} /$ session), SDH ( $\geq 5$ sessions/week, 2-4 h/session) or NHD ( $\geq 4$ sessions/week, $\geq 5 \mathrm{~h} /$ session, occurring at night).

\section{Comorbidities}

Comorbidities were defined as follows: (1) coronary artery disease, i.e. coronary stenosis documented by angiography or a history of myocardial infarction or previous coronary revascularization (angioplasty, stenting or bypass surgery); (2) peripheral vascular disease, i.e. a history of lower extremity revascularization or digit or extremity amputation or a history of claudication and ischemic extremity changes or gangrene; (3) cerebrovascular disease, i.e. a stroke or transient ischemic attack documented by computed tomography scanning, magnetic resonance imaging or classic clinical signs and symptoms with confirmation by a neurologist, or when the diagnosis had been noted in the medical records at least twice by 2 different physicians; (4) congestive heart failure, i.e. classic signs and symptoms and documentation from echocardiography or chest X-ray or complete symptom resolution with ultrafiltration, and (5) diabetes, i.e. a history of hypoglycemic agent or insulin use [26].

\section{Statistical Analysis}

We used SAS version 9.2 (SAS Institute Inc., Cary, N.C., USA) for all analyses. We compared baseline characteristics using analysis of variance or the Kruskal-Wallis test for continuous variables, depending on the distribution, and $\chi^{2}$ tests for categorical variables. Patients were followed for infectious complications from January 1, 2000, through December 31, 2010. We calculated the person-time incidence rate as the number of infectious events per total number of fistula days (standardized to 1,000 fistula days). We compared the rate of infectious events between the groups using the exact binomial test. Statistical significance was defined as a $p$ value $\leq 0.05$. 
Fig. 1. Use of the two cannulation techniques in the different types of hemodialysis.

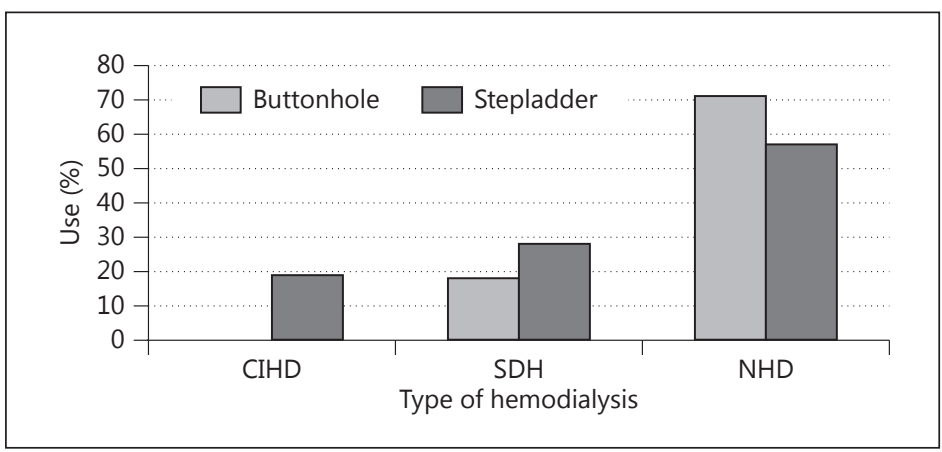

Table 1. Patient characteristics

\begin{tabular}{|c|c|c|c|c|}
\hline & \multicolumn{3}{|c|}{ Dialysis frequency } & \multirow[t]{2}{*}{$\mathrm{p}$} \\
\hline & $\begin{array}{l}\text { CIHD } \\
(n=457)\end{array}$ & $\begin{array}{l}\text { SDH } \\
(n=46)\end{array}$ & $\begin{array}{l}\text { NHD } \\
(n=128)\end{array}$ & \\
\hline Mean age \pm SD, years & $58.8 \pm 14.9$ & $48.9 \pm 14.2$ & $43.1 \pm 13.2$ & 0.01 \\
\hline Age $\geq 65$ years & $168(36.8 \%)$ & $5(10.9 \%)$ & $5(3.9 \%)$ & 0.08 \\
\hline Male & $263(57.5 \%)$ & $35(76 \%)$ & $69(54 \%)$ & 0.0085 \\
\hline Caucasian & $346(75.7 \%)$ & $36(78.3 \%)$ & $88(68.8 \%)$ & 0.32 \\
\hline Primary renal diagnosis & & & & 0.99 \\
\hline Diabetes & $114(25.0 \%)$ & $7(15.2 \%)$ & $19(14.8 \%)$ & \\
\hline Hypertension & $61(13.4 \%)$ & $3(6.5 \%)$ & $10(7.8 \%)$ & \\
\hline Glomerulonephritis & $93(20.4 \%)$ & $22(47.8 \%)$ & $58(45.3 \%)$ & \\
\hline Interstitial nephritis & $7(1.5 \%)$ & $1(2.17 \%)$ & $3(2.3 \%)$ & \\
\hline Hereditary/other (includes diabetes and hypertension) & $112(24.5 \%)$ & $11(23.9 \%)$ & $33(25.8 \%)$ & \\
\hline Unknown & $47(10.3 \%)$ & $2(4.4 \%)$ & $5(3.9 \%)$ & \\
\hline \multicolumn{5}{|l|}{ Comorbidities } \\
\hline Diabetes & $160(36.0 \%)$ & $9(19.6 \%)$ & $29(22.7 \%)$ & 0.66 \\
\hline Hypertension & $313(68.5 \%)$ & $42(91.3 \%)$ & $105(82.0 \%)$ & 0.14 \\
\hline Coronary artery disease & $133(29.1 \%)$ & $12(26.1 \%)$ & $15(11.7 \%)$ & 0.02 \\
\hline Congestive heart failure & $73(16.0 \%)$ & $11(23.9 \%)$ & $10(7.8 \%)$ & 0.004 \\
\hline Peripheral vascular disease & $42(9.2 \%)$ & $4(8.7 \%)^{\mathrm{a}}$ & $11(8.6 \%)$ & 0.98 \\
\hline Cerebrovascular disease & $43(9.4 \%)$ & $4(8.7 \%)$ & $4(3.1 \%)$ & 0.12 \\
\hline
\end{tabular}

CIHD: 3 sessions/week, $\leq 4 \mathrm{~h} /$ session; SDH: $\geq 5$ sessions/week, 2-4 h/session; NHD: $\geq 4$ sessions/week, $\geq 5$ h/session. Variables were compared using t or $\chi^{2}$ tests; $p$ values for SDH vs. NHD only. There were statistical differences in patient characteristics between those who cannulated with the buttonhole technique and those using the stepladder technique (see table 2) in patients performing frequent hemodialysis. ${ }^{\text {a }}$ Conventional cohort.

\section{Results}

In the frequent dialysis cohort, $46(26.4 \%)$ received SDH and $128(73.6 \%)$ received NHD. The buttonhole technique was more commonly used by those receiving NHD (72\%) versus SDH (50\%; fig. 1). Overall, the buttonhole technique was used on 198,910 fistula days, while stepladder cannulation was used on 99,681 fistula days for the frequent dialysis cohort. All patients on CIHD used the stepladder technique for a total of 405,174 fistula days.

The patient characteristics at the time of starting CIHD - or, if they were in the frequent dialysis cohort, at the time the patient switched to frequent dialysis - are summarized in table 1. Overall, the patients on frequent dialysis were an average of 46 years old (range: 
Lok et al.: Frequent Hemodialysis Fistula Infectious Complications

Table 2. Frequent hemodialysis patient characteristics according to the cannulation technique

\begin{tabular}{|c|c|c|c|}
\hline & \multicolumn{2}{|c|}{ Cannulation technique } & \multirow[t]{2}{*}{$\mathrm{p}$} \\
\hline & $\begin{array}{l}\text { buttonhole } \\
(\mathrm{n}=89)\end{array}$ & $\begin{array}{l}\text { stepladder } \\
(\mathrm{n}=104)\end{array}$ & \\
\hline Mean age \pm SD, years & $43 \pm 12.1$ & $48 \pm 15.7$ & 0.01 \\
\hline Age $\geq 65$ years & $2(2.3 \%)$ & $13(12.5 \%)$ & 0.008 \\
\hline Male & $55(61.8 \%)$ & $61(58.7 \%)$ & 0.66 \\
\hline Caucasian & $67(75.2 \%)$ & $70(67.3 \%)$ & 0.61 \\
\hline Primary renal diagnosis & & & $0.002^{\mathrm{a}}$ \\
\hline Diabetes & $4(4.5 \%)$ & $26(25.0 \%)$ & \\
\hline Hypertension & $8(9.0 \%)$ & $9(8.7 \%)$ & \\
\hline Glomerulonephritis & $42(47.2 \%)$ & $42(40.4 \%)$ & \\
\hline Interstitial nephritis & $3(3.4 \%)$ & $2(1.9 \%)$ & \\
\hline Hereditary/other (includes diabetes and hypertension) & $28(31.5 \%)$ & $20(19.2 \%)$ & \\
\hline Unknown & $4(4.5 \%)$ & $5(4.8 \%)$ & \\
\hline \multicolumn{4}{|l|}{ Comorbidities } \\
\hline Diabetes & $9(10.1 \%)$ & $38(36.5 \%)$ & $<0.001$ \\
\hline Hypertension & $75(84.3 \%)$ & $88(84.6 \%)$ & 0.95 \\
\hline Coronary artery disease & $3(3.4 \%)$ & $31(29.8 \%)$ & $<0.001$ \\
\hline Congestive heart failure & $7(7.8 \%)$ & $16(15.4 \%)$ & 0.12 \\
\hline Peripheral vascular disease & $1(1.1 \%)$ & $18(17.3 \%)$ & $<0.002$ \\
\hline Cerebrovascular disease & $2(2.3 \%)$ & $8(7.7 \%)$ & 0.11 \\
\hline
\end{tabular}

a Differences primarily due to diabetes/diabetes plus hypertension as the cause of end-stage kidney disease in patients using the stepladder technique.

20-69 years); $60 \%$ were male and $71 \%$ were Caucasian; $22 \%$ had diabetes and $84 \%$ had hypertension. The patients on NHD were slightly younger than those on SDH. More patients on SDH had coronary heart disease and heart failure (table 1). Frequent hemodialysis patients cannulating with the buttonhole cannulation technique were younger and had less comorbidity (table 2). Those who cannulated using the stepladder technique more often had diabetes as a cause of end-stage kidney disease and as a comorbidity and more often had vascular disease (coronary artery disease and peripheral vascular disease).

\section{Infectious Complications}

Infectious complications are summarized in table 3 . There were 39 buttonhole-related bacteremias and at least 2 local buttonhole site infections. The buttonhole bacteremia rate was $0.196 / 1,000$ fistula days. S. aureus accounted for $85 \%$ of these. There were 5 related hospitalizations and 3 metastatic infections (endocarditis, septic arthritis, mycotic aneurysm of a left brachiocephalic fistula and loss of fistula). No fistula-related infections were documented for the patients using stepladder cannulation at home. In comparison, there was only a single case of possible fistula-related infection among the patients using stepladder cannulation; this patient was receiving CIHD (rate: 0.002/1,000 fistula days). Of those using the buttonhole technique, $19.3 \%$ required buttonhole retraining. On average, this retraining took 5.88 days. There was no difference in the mean number of days required for buttonhole retraining between the SDH group ( $5.33 \pm 2.3$ days) and the NHD group (6.0 \pm 5.9 days). 
Lok et al.: Frequent Hemodialysis Fistula Infectious Complications

Table 3. Infectious complications: buttonhole versus stepladder cannulation

\begin{tabular}{|c|c|c|c|c|}
\hline & \multicolumn{3}{|c|}{ Cannulation technique } & \multirow[t]{2}{*}{$\mathrm{p}$} \\
\hline & $\begin{array}{l}\text { buttonhole } \\
(198,910 \\
\text { fistula days })\end{array}$ & $\begin{array}{l}\text { stepladder, } \\
\text { in center } \\
(405,174 \\
\text { fistula days) }\end{array}$ & $\begin{array}{l}\text { stepladder, } \\
\text { home dialysis } \\
(99,681 \\
\text { fistula days) }\end{array}$ & \\
\hline Bacteremia events & 39 & 0 & 0 & \\
\hline S. aureus & $85 \%$ & $\mathrm{~N} / \mathrm{A}$ & $\mathrm{N} / \mathrm{A}$ & \\
\hline Site infections & 2 & 1 & 0 & \\
\hline Metastatic infections & 3 & 0 & 0 & \\
\hline \multicolumn{5}{|c|}{ Person-time incidence rate (per 1,000 fistula days) } \\
\hline Bacteremia rate & 0.20 & 0.000 & 0.000 & $<0.0001$ \\
\hline Non-bacteremia infectious rate & 0.01 & 0.002 & 0.000 & $<0.0001$ \\
\hline Overall infectious rate & 0.21 & 0.002 & 0.000 & $<0.0001$ \\
\hline
\end{tabular}

\section{Discussion}

A functioning vascular access is required for hemodialysis. However, each access type is associated with different frequencies of complications. A fistula, once established, is associated with the lowest risk of complications, including infections, such that all current renal guidelines recommend the use of a fistula over a graft or catheter, if possible. However, for patients who are treated at home, the fistula may create additional challenges compared to a graft or catheter. Self-cannulation with a fistula may be more difficult than with a graft, especially if cannulation areas are limited. In response to this problem, buttonhole or same-site cannulation was introduced in the 1970s and is used by many patients treated with home hemodialysis. However, our study shows that the risk of infection with the buttonhole cannulation technique for patients undergoing more frequent dialysis at home is significantly increased compared to that for patients using the stepladder technique. Similarly, the risk of infection with the buttonhole technique at home is much higher than that with the stepladder technique used for patients treated in-center and at home (table 3).

Our results are consistent with other studies in which the buttonhole technique was used for patients treated with in-center CIHD and for patients treated at home with more frequent hemodialysis. As part of a quality assurance project, Labriola et al. [27] reported an increase in infections with buttonhole cannulation for patients treated with in-center CIHD from a baseline of $0.17 / 1,000$ fistula days when stepladder cannulation was used to $0.43 / 1,000$ fistula days with buttonhole cannulation. Complicated infectious events occurred in 12 patients. Although the infection risk was reduced to $0.34 / 1,000$ fistula days after the implementation of an infection prevention program, an increased infection risk persisted [27]. Similarly, an increased risk of infections was reported in a randomized controlled trial comparing buttonhole with stepladder cannulation for patients being treated with in-center CIHD [28]. For patients treated with NHD at home, 10 episodes of $S$. aureus bacteremia were documented over 93.4 patient-months of follow-up with the buttonhole technique $0.32 / 1,000$ fistula days) [29]. Four of the 10 patients suffered serious metastatic infections, including 1 death from sepsis. The infection risk was substantially but not completely eliminated with the addition of mupirocin cream at the buttonhole site after dialysis secondary to nonadherence $(0.03 / 1,000$ fistula days). The rate remained substantially higher than the $0.005 / 1,000$ fistula days for patients treated with in-center CIHD using stepladder cannulation [29]. These results are also supported by the Australian experience, with a documented increased risk of hospital 
admissions for vascular access infections in NHD patients using buttonhole cannulation, the majority of which were due to $S$. aureus [24]. Contrary to the above studies, one randomized controlled trial of buttonhole versus stepladder cannulation in CIHD patients evaluated vascular access outcomes and did not show an increased risk of infections by the cannulation technique. Another randomized trial evaluating frequent hemodialysis compared with conventional hemodialysis also did not find an increased infection risk; however, the vascular access and cannulation outcomes were secondary findings. The former study suffers from a relatively high dropout rate, and both studies have small numbers of patients, using buttonhole cannulation followed up for a short period of time, increasing the risk of a type ll error $[30,31]$.

In the above studies in which the infection risk was increased, it remained unclear if the problem was related only to the buttonhole technique versus the cannulator (health care professional vs. self), site (home vs. center) or frequency (conventional vs. daily). Our study suggests that the frequency of cannulation is not the issue, as our infection risk with stepladder cannulation with frequent dialysis at home was essentially nonexistent and comparable to our in-center CIHD access infection rate. Also, all of the frequent home hemodialysis patients included in our study were self-cannulators, suggesting that self-cannulation is unlikely the issue. Lastly, patients with greater comorbidity and in a potentially more immunocompromised state, as may occur with diabetes, may be at greater risk of infection. However, we found that patients in the buttonhole cannulation group were younger and had less comorbidity than those cannulating with the stepladder technique.

There are a number of limitations to our study, including the observational design. We cannot guarantee that we have not missed any superficial fistula infections as these are not tracked by the vascular access team or the infection control nurse. The strengths of our study include the prospective data collection, the large number of fistula days and the inclusion of two different dialysis centers. Our study also includes a frequent hemodialysis control group that did not use buttonhole cannulation that addresses the issues of frequency and of the type of cannulator. In terms of our future directions, we are currently conducting a multicenter controlled pilot trial of buttonhole versus stepladder cannulation for patients who perform home hemodialysis. In addition to our primary feasibility outcome, other important outcomes include determining if buttonhole cannulation is associated with reduced clinical complications and costs compared with stepladder cannulation in intensive home hemodialysis patients.

In conclusion, buttonhole cannulation is associated with an increased risk of bacteremias, the majority of which are due to $S$. aureus with potentially devastating consequences. Until further studies become available, the use of buttonhole cannulation should be limited to patients with short usable fistula segments or those who would otherwise be unable to selfcannulate. In these circumstances, strict infection control measures must be followed.

\section{Disclosure Statement}

The authors declare no conflicts of interest related to the manuscript.

\section{References}

1 Lok CE, Sontrop JM, Tomlinson G, Rajan D, Cattral M, Oreopoulos G, Harris J, Moist L: Cumulative patency of contemporary fistulas versus grafts (2000-2010). Clin J Am Soc Nephrol 2013;8:810-818.

$\checkmark 2$ Lok CE, Foley R: Vascular access morbidity and mortality: trends of the last decade. Clin J Am Soc Nephrol 2013;8:1213-1219. 
Lok et al.: Frequent Hemodialysis Fistula Infectious Complications

- 3 Huijbregts HJ, Bots ML, Wittens CH, Schrama YC, Moll FL, Blankestijn PJ: Hemodialysis arteriovenous fistula patency revisited: results of a prospective, multicenter initiative. Clin J Am Soc Nephrol 2008;3:714-719.

$\checkmark 4$ KDOQI clinical practice guidelines for vascular access. Am J Kidney Dis 2006;48:S176-S247.

5 Tonnessen BH, Money SR: Embracing the fistula first national vascular access improvement initiative. J Vasc Surg 2005;42:585-586.

6 http://www.fistulafirst.org (accessed September 9, 2011).

7 Canadian Society of Nephrology (CSN): Report of the Canadian Society of Nephrology vascular access working group. Semin Dial 2012;25:22-25.

-8 Tordoir J, Canaud B, Haage P, Konner K, Basci A, Fouque D, Kooman J, Martin-Malo A, Pedrini L, Pizzarelli F, Tattersall J, Vennegoor M, Wanner C, ter Wee P, Vanholder R: EBPG on vascular access. Nephrol Dial Transplant 2007;22(suppl 2):ii88-ii117.

-9 Allon M, Robbin ML: Increasing arteriovenous fistulas in hemodialysis patients: problems and solutions. Kidney Int 2002;62:1109-1124.

$\$ 10$ Lee T, Barker J, Allon M: Needle infiltration of arteriovenous fistulae in hemodialysis: risk factors and consequences. Am J Kidney Dis 2006;47:1020-1026.

11 Mott S: Considerations at initiation of cannulation. Nephrol News Issues 2013;27:26, 28.

12 Bay WH, Van Cleef S, Owens M: The hemodialysis access: preferences and concerns of patients, dialysis nurses and technicians, and physicians. Am J Nephrol 1998;18:379-383.

13 Chaudhry M, Bhola C, Joarder M, Zimmerman D, Quinan P, Mendelssohn D, Lok CE: Seeing eye to eye: the key to reducing catheter use. J Vasc Access 2011;12:120-126.

14 Twardowski ZJ: Constant site (buttonhole) method of needle insertion for hemodialysis. Dial Transplant 1995; 24:559-560, 576.

15 Verhallen AM, Kooistra MP, van Jaarsveld BC: Cannulating in haemodialysis: rope-ladder or buttonhole technique? Nephrol Dial Transplant 2007;22:2601-2604.

-16 Marticorena RM, Hunter J, Macleod S, Petershofer E, Dacouris N, Donnelly S, Goldstein MB: The salvage of aneurysmal fistulae utilizing a modified buttonhole cannulation technique and multiple cannulators. Hemodial Int 2006;10:193-200.

-17 Marticorena RM, Hunter J, Macleod S, Petershofer E, Kashani M, De la Cruz J, Cook R, Dacoiris N, McFarlane PA, Goldstein MB, Donnelly SM: Use of the BioHole ${ }^{\mathrm{TM}}$ device for the creation of tunnel tracks for buttonhole cannulation of fistula for hemodialysis. Hemodial Int 2011;15:243-249.

18 Ball LK, Treat L, Riffle V, Scherting D, Swift L: A multi-center perspective of the buttonhole technique in the Pacific Northwest. Nephrol Nurs J 2007;34:234-241.

19 Hartig V, Smyth W: 'Everyone should buttonhole': a novel technique for a regional Australian renal service. J Ren Care 2009;35:114-119.

-20 Pergolotti A, Rich E, Lock K: The effect of the buttonhole method versus the traditional method of AV fistula cannulation on hemostasis, needle stick pain, pre-needle stick anxiety, and presence of aneurysms in ambulatory patients on hemodialysis. Nephrol Nurs J 2011;38:333-336.

21 Ward J, Shaw K, Davenport A: Patients' perspectives of constant-site (buttonhole) cannulation for haemodialysis access. Nephron Clin Pract 2010;116:c123-c127.

-22 Hashmi A, Cheema MQ, Moss AH: Hemodialysis patients' experience with and attitudes toward the buttonhole technique for arteriovenous fistula cannulation. Clin Nephrol 2010;74:346-350.

23 van Loon MM, Goovaerts T, Kessels AG, van der Sande FM, Tordoir JH: Buttonhole needling of haemodialysis arteriovenous fistulae results in less complications and interventions compared to the rope-ladder technique. Nephrol Dial Transplant 2010;25:225-230.

24 Van Eps CL, Jones M, Ng T, Johnson DW, Campbell SB, Isbel NM, Mudge DW, Beller E, Hawley CM: The impact of extended-hours home hemodialysis and buttonhole cannulation technique on hospitalization rates for septic events related to dialysis access. Hemodial Int 2010;14:451-463.

25 Ludlow V: Buttonhole cannulation in hemodialysis: improved outcomes and increased expense - is it worth it? CANNT J 2010;20:29-37.

26 Lok CE, Allon M, Moist L, Oliver MJ, Shah H, Zimmerman D: Risk equation determining unsuccessful cannulation events and failure to maturation in arteriovenous fistulas (REDUCE FTM I). J Am Soc Nephrol 2006;17: 3204-3212.

-27 Labriola L, Crott R, Desmet C, Andre G, Jadoul M: Infectious complications following conversion to buttonhole cannulation of native arteriovenous fistulas: a quality improvement report. Am J Kidney Dis 2011;57:442448.

28 MacRae JM, Ahmed SB, Atkar R, Hemmelgarn BR: A randomized trial comparing buttonhole with rope ladder needling in conventional hemodialysis patients. Clin J Am Soc Nephrol 2012;7:1632-1638.

-29 Nesrallah GE, Cuerden M, Wong JH, Pierratos A: Staphylococcus aureus bacteremia and buttonhole cannulation: long-term safety and efficacy of mupirocin prophylaxis. Clin J Am Soc Nephrol 2010;5:1047-1053.

30 Vaux E, King J, Lloyd S, Moore J, Bailey L, Reading I, Naik R: Effect of buttonhole cannulation with a polycarbonate peg on in-center hemodialysis fistula outcomes: a randomized controlled trial. Am J Kidney Dis 2013; 62:81-88.

31 Suri RS, Larive B, Sherer S, Eggers P, Gassman J, James SH, Lindsay RM, Lockridge RS, Ornt DB, Rocco MV, Ting GO, Kliger AS: Risk of vascular access complications with frequent hemodialysis. J Am Soc Nephrol 2013;24: 498-505. 